\title{
Correlação entre religiosidade, espiritualidade e qualidade de vida: uma revisão de literatura
}

\section{Correlation between religiousness, spirituality and quality of life: a review of literature}

\section{Correlación entre la religiosidad, la espiritualidad y calidad de vida: una revisión de la literatura}

\author{
Cynthia de Freitas Melo* \\ Universidade de Fortaleza - UNIFOR, Fortaleza, Ceará, Brasil
}

\section{Israel Silva Sampaio**}

Universidade Estadual do Ceará - UECE, Fortaleza, Ceará, Brasil

Deborah Leite de Abreu Souza***

Universidade Estadual do Ceará - UECE, Fortaleza, Ceará, Brasil

Nilberto dos Santos Pinto****

Universidade Estadual do Ceará - UECE, Fortaleza, Ceará, Brasil

\begin{abstract}
RESUMO
O fenômeno da religiosidade, por tanto tempo visto no meio científico através de olhares críticos, tem ganhado espaço nos estudos sobre saúde, em especial na Psicologia. Sob essa perspectiva, a presente pesquisa objetivou realizar uma revisão bibliográfica de metanálise, verificando os estudos existentes que exploram a correlação entre a qualidade de vida e a religiosidade, em diferentes aspectos e contextos. Seguindo uma rígida sequência de passos planejados no método, utilizou-se como descritores os termos "religiosidade", "qualidade de vida" e "espiritualidade", sendo coletados 263 artigos de língua portuguesa ou inglesa disponíveis em todos os periódicos indexados no portal Capes. Após verificação dos critérios de inclusão e exclusão de todo o material, foram analisados 14 documentos. Os resultados mostraram que a religiosidade e a espiritualidade têm correlação com a qualidade de vida, atuando principalmente como enfrentamento em situações adversas. Assim, fica evidente a importância desses fenômenos para as práticas de saúde, ressaltando-se a necessidade de valorizá-los e incluí-los nas formações profissionais.
\end{abstract}

Palavras chaves: qualidade de vida, religiosidade, espiritualidade, metanálise.

\section{ABSTRACT}

The phenomenon of religion, so long seen in the scientific community through critical eyes, has gained ground in health studies, particularly in psychology. From this perspective, the present study aimed to perform a 
literature review of meta-analysis verifying existing studies that explored the correlation between quality of life and religiosity in different ways and contexts. Following a rigid sequence of steps planned in the method, was used as descriptors the terms "religion", "quality of life" and "spirituality", and listed 263 items Portuguese and English available in all journals indexed in Capes portal. After verification of inclusion criteria and exclusion of all material, 14 documents were analyzed. The results showed that religiousness and spirituality are correlated with quality of life, working mainly as coping in adverse situations. Thus, it is evident the importance of these phenomena for practical health, emphasizing the need to value them and include them in vocational training courses.

Keywords: quality of life, religiosity, spirituality, meta-analysis.

\section{RESUMEN}

El fenómeno de la religión, siempre se ve en la comunidad científica a través de los ojos críticos, ha ganado terreno en los estudios de salud, sobre todo en la psicología. Desde esta perspectiva, el presente estudio tuvo como objetivo realizar una revisión bibliográfica de los meta-análisis de la verificación de los estudios existentes que exploraron la relación entre calidad de vida y la religiosidad de diferentes formas y contextos. Tras una secuencia rígida de pasos planificados en la metodología, se utilizó como descriptores los términos "religión", "calidad de vida" y "espiritualidad", y citadas 263 artículos en portugués e inglés disponibles en todas las revistas indexadas en el portal de Capes. Después de la verificación de los criterios de inclusión y exclusión de todos los materiales, se analizaron 14 documentos. Los resultados mostraron que la religiosidad y la espiritualidad están relacionadas con la calidad de vida, trabajando principalmente como hacer frente a situaciones adversas. Por lo tanto, es evidente la importancia de estos fenómenos para la salud la práctica, haciendo hincapié en la necesidad de valorarlos e incluirlos en los cursos de formación profesional.

Palabras clave: calidad de vida, la religiosidad, espiritualidad, metaanálisis.

\section{Introdução}

Por um longo período, ciência e espiritualidade/religiosidade eram vistas como áreas totalmente distintas e opostas. Atualmente, ainda predominam no âmbito da ciência, concepções racionalistas e mecanicistas que tendem a desvalorizar aquilo que não pode ser totalmente mensurado, previsto ou controlado pela tecnologia e pela razão. Reforça-se, todavia, que em contraposição, existem linhas teóricas que buscam promover uma compreensão mais adequada desses fenômenos, contribuindo com meios eficazes de analisá-los e estudá-los (Fleck, Borges, Bolognesi, \& Rocha, 2003).

Nesse contexto, o fenômeno da religiosidade tem sido alvo de diversas discussões e pesquisas nos mais variados campos científicos. No que se refere ao campo da saúde e, mais especificamente, ao da Psicologia, esse fenômeno encontrava-se, inicialmente, relacionado ao desenvolvimento de psicopatologias, tendo em vista que as doutrinas religiosas eram consideradas alienantes e repressoras da 
sexualidade (Almeida, 2009). No entanto, pesquisas recentes apontam a religiosidade e a espiritualidade como alguns dos aspetos mais significativos da subjetividade humana, observando que eles se relacionam com a construção de sentido e ordenação de vida dos indivíduos, influenciando também a sua saúde de forma positiva.

Dentre essas pesquisas, destacam-se nas últimas duas décadas, os estudos sobre as relações entre religiosidade, espiritualidade e saúde, em expansão na literatura médica e psicológica. Segundo esses estudos, maiores níveis de envolvimento religioso estão associados positivamente com indicadores que contribuem para o bem-estar psicológico, como felicidade, satisfação com a vida, afeto positivo e moral elevado (Stroppa \& Moreira-Almeida, 2008). Nesse contexto, destacam-se, também, diversos estudos que apontam a correlação entre religiosidade e qualidade de vida geral (Barricelli et al., 2012, Rocha \& Fleck, 2011; Medeiros, 2010).

Neste sentido, o presente estudo objetivou investigar a relação entre religiosidade, espiritualidade e a percepção de qualidade de vida em diferentes contextos. Deste modo, pretende-se contribuir para 0 desenvolvimento de trabalhos que tratem da temática abordada, bem como verificar de forma mais específica a importância dessa relação para os pacientes portadores de doenças crônicas, os idosos, pacientes com câncer, portadores de HIV, pessoas em situação de luto, estudantes universitários e pessoas com depressão.

\subsection{Qualidade de vida: aspectos conceituais e teóricos}

Inicialmente, logo após a Segunda Guerra Mundial, o conceito de qualidade de vida estava diretamente relacionado a variáveis econômicas capitalistas, levando-se em consideração a obtenção de bens materiais para avaliar o nível de qualidade de vida dos sujeitos. Aos poucos o conceito foi sendo ampliado e passou a integrar também outros aspectos sociais, como educação, saúde e lazer; estando inclusive intimamente ligado ao movimento da promoção de saúde, iniciado em 1974, no Canadá (Kluthcovsky \& Takayanagui, 2007).

Atualmente, o conceito de qualidade de vida tem se tornado bastante significativo para a sociedade em geral e sua utilização perpassa desde campanhas publicitárias até pesquisas científicas nas mais diversas áreas (Seidl \& Zannon, 2004). No campo da saúde, com o crescente avanço tecnológico e a consequente desumanização das práticas de cuidado, a discussão acerca da qualidade de vida se mostra ainda mais importante (Kluthcovsky \& Takayanagui, 2007). A partir da ampliação do conceito de saúde, defendida pela Organização Mundial de Saúde (OMS) como o "estado de completo bem-estar físico, mental e social", já se tornou comum entre os profissionais da área a afirmação de que saúde está mais relacionada com o conceito 
de qualidade de vida do que apenas com a ausência de doença ou enfermidade (Segre \& Ferraz, 1997).

Porém, apesar de buscar o rompimento com o paradigma biomédico, as práticas em saúde ainda não têm como finalidade a promoção de um conceito ampliado de saúde, limitando-se a práticas tradicionais, e sem um compromisso real com a qualidade de vida dos sujeitos (Minayo, 2000). Ainda assim, diversas tentativas vêm sendo feitas a fim de construir um conceito mais dinâmico de saúde e nesse contexto o conceito de qualidade de vida se torna essencial, levando em consideração que se trata de um conceito que só é possível de ser avaliado pelo próprio sujeito (Segre \& Ferraz, 1997).

Em relação à qualidade de vida, é importante ressaltar algumas premissas para a sua compreensão, as quais são:

a) Subjetividade - a percepção do indivíduo, ou seja, a perspectiva do indivíduo que realmente conta, sendo um conceito intrínseco em que o próprio sujeito participa da sua definição (Seidl \& Zannon, 2004);

b) Multidimensionalidade - o indivíduo passa a ser visto como um ser multidimensional em que vários aspectos vão estar envolvidos na sua relação consigo mesmo e com o mundo, influenciando para a sua qualidade de vida (Seidl \& Zannon, 2004);

c) Bipolaridade - consiste no reconhecimento de características que devem estar presentes e ausentes para que a qualidade de vida seja considerada satisfatória (Fleck, 2008);

d) Mutabilidade - leva em consideração que a avaliação da qualidade de vida pode variar de acordo com o tempo, o local e o contexto cultural no qual está inserido (Paschoal, 2001).

Diante de tais aspectos, torna-se evidente que a noção de qualidade de vida propõe uma quebra de paradigmas, buscando englobar elementos complexos e difíceis de serem mensurados. Além disso, podem contribuir com a construção de uma clínica ampliada e com o conceito de saúde biopsicossocial, onde são ressaltados os aspectos socioeconômicos, psicológicos e culturais, que são essenciais nos processos de prevenção, promoção e reabilitação da saúde (Seidl \& Zannon, 2004).

Com isso, evidencia-se a noção interdisciplinar do conceito de qualidade de vida, devendo conciliar os saberes de diversas áreas do conhecimento no intuito de compreender o indivíduo na sua complexidade e em suas particularidades (Seidl \& Zannon, 2004). Assim, é interessante avaliar todos os aspectos envolvidos na qualidade de vida e, consequentemente, com a saúde dos sujeitos. Atualmente, as pesquisas têm demonstrado o fenômeno da religiosidade como um fator influenciador da qualidade de vida dos sujeitos, ressaltando a importância de sua compreensão (Almeida, 2009). 


\subsection{Religiosidade, espiritualidade e suas implicações com a saúde}

O fenômeno da religiosidade tem sido alvo de diversos estudos nos mais variados campos científicos. Sabe-se que as manifestações religiosas estão presentes na humanidade desde os primórdios de sua existência e, ainda nos dias de hoje, permanecem muito presentes na vida da maioria das pessoas (Almeida, 2009). Sendo assim, não é de se espantar o interesse dos pesquisadores em identificar como esse fenômeno atua na dinâmica social dos indivíduos, influenciando o seu comportamento, a sua concepção de si mesmo, do outro, do mundo que o rodeia e até mesmo a sua saúde (Sanchez \& Nappo, 2007; Alminhana \& Moreira-Almeida, 2008; Alves, 2010).

Ao abordar a concepção de religiosidade Vergote e Gandelman (2013) afirmam que a religião é um fenômeno relativo ao desejo, ou seja, o homem tem consciência de sua finitude e limitações, mas tem 0 desejo de superá-la através de experiências e modos de existência que lhe fazem celebrar e gozar. Sendo assim, a religiosidade aparece então como um fenômeno que está relacionado com a busca do homem por responder os seus questionamentos existenciais e dá sentido a sua vida.

Segundo Angerami-Camon (2008), a religiosidade consiste na busca do homem por transcendência e é um dos meios pelos quais ele pode vivenciar a sua espiritualidade. O autor afirma ainda que a religiosidade faz com que o homem reflita sobre si mesmo e as suas relações, procurando significados para a sua existência que estão além do mundo objetivo. Sendo assim, a religiosidade é algo que constantemente está presente nas psicoterapias e o psicólogo deve lidar com o tema a partir da importância e significado que possui para o paciente, para que este não seja encarado como alienado ou a religiosidade como algo prejudicial, de forma preconcebida (Angerami-Camon, 2004).

A religiosidade, na maioria das vezes, não precisa de comprovação empírica para que possa ser vivenciada, fazendo com que essas duas linhas de conhecimento sejam consideradas opostas (Motta \& Júnior, 2011). No entanto, para a compreensão psicológica dos fenômenos religiosos essa discussão se torna inútil, pois se a religiosidade consistir em algo estimado pelo indivíduo terá implicações em sua vida concreta, independente da veracidade de suas crenças ou dogmas (James, 1985).

Atualmente, para se definir o conceito de religião é importante também diferenciá-lo do conceito de espiritualidade, já que são construtos que estão intimamente ligados e muitas vezes são considerados sinônimos. Para Murakami e Campos (2012), a espiritualidade se constitui de um sentimento íntimo existencial, uma 
busca pelo sentido de viver e estar no mundo e que não necessariamente vai estar ligado à crença em algo maior, como um Deus. Já a religiosidade, pode ser entendida como sendo um conjunto de crenças e práticas pertencentes a uma doutrina, que são compartilhadas e seguidas por um grupo de pessoas, através de cultos ou rituais que envolvem necessariamente a noção de fé (Murakami \& Campos, 2012).

Gordon Allport, interessado pela temática da religião, como derivado de seus estudos sobre a personalidade, especialmente na sua relação com a dimensão moral, criou a classificação mais utilizada no meio acadêmico para a orientação religiosa, distribuídas em dois tipos: a intrínseca e a extrínseca. Na religiosidade intrínseca o indivíduo apresenta uma fé bem amadurecida, procurando viver realmente de acordo com os princípios doutrinários em que acredita, harmonizando suas necessidades e interesses às suas crenças, esforçando-se por internalizá-las e apresentando um relacionamento saudável com a sua religião, já que o princípio motivador de suas atitudes se encontra na própria religião, que atribui significado à sua vida. Já na religiosidade extrínseca, a religião é utilizada como um meio para atingir outros fins, benefícios exteriores (de status, segurança e distração), de modo que a pessoa se volta ao sagrado ou a Deus, mas sem desapegar-se do self, como, por exemplo, os indivíduos que se convertem a uma determinada religião para ampliar os seus relacionamentos sociais, devido a um relacionamento afetivo ou até mesmo como uma manobra de publicidade (Murakami \& Campos, 2012). Observa-se, portanto, que, como afirmava Allport, ao estabelecer uma comparação entre as duas orientações: os extrínsecos usam sua religião enquanto os intrínsecos a vivenciam (Alminhana \& Moreira-Almeida, 2009).

Muitas pesquisas apontam que a religiosidade pode contribuir com aspectos favoráveis ou não à saúde dos indivíduos. Atualmente, muitos estudiosos argumentam que a religiosidade gera níveis patológicos de culpa, diminui a autoestima e possui ideologias voltadas para a repressão da raiva e das manifestações sexuais (Medeiros, 2010).

Em contrapartida, outros estudiosos defendem que o envolvimento religioso reduz a ansiedade existencial ao oferecer respostas que permitem uma organização de conflitos emocionais, estabelecem um sistema de orientação moral e ética, além de desestimular práticas consideradas destrutivas para a saúde de uma forma geral (Medeiros, 2010).

Um fator importante a ser mencionado é que, segundo Antoniazzi (2003), 93,7\% dos brasileiros se consideram religiosos, dentre os quais $25 \%$ afirmam estar envolvidos com mais de uma religião. Apenas $7,3 \%$ se declararam sem religião, observou-se, todavia que muitos desses afirmam crer em alguma Divindade ou Ser Superior, 
demonstrando uma forma de espiritualidade que não possui vinculação religiosa (Antoniazzi, 2003).

Também existem evidências crescentes de que a religiosidade está associada com saúde mental. Uma revisão de 200 artigos demonstrou que em $50 \%$ dos casos ela está associada com fatores positivos e em $25 \%$ com fatores negativos. Nesta revisão a religiosidade apareceu como sendo um fator protetor para suicídio, abuso de drogas e álcool, comportamento delinquente, satisfação marital, sofrimento psicológico e alguns diagnósticos de psicoses funcionais (Rocha \& Fleck, 2004).

Diante do que foi exposto, torna-se intrigante explorar a produção da literatura hoje sobre a relação entre religiosidade, espiritualidade e qualidade de vida através de-um procedimento metodológico rígido de revisão de literatura de metanálise.

\section{Método}

\subsection{Tipo de estudo}

Para a concretização do estudo, realizou-se uma revisão da literatura, tipo de pesquisa caracterizada pela análise da produção bibliográfica em determinada área temática, situada em um período específico, oferecendo uma visão geral sobre a temática estudada e evidenciando novas ideias ou os temas que têm recebido maior ou menor ênfase na literatura selecionada (Noronha \& Ferreira, 2000). Dentre os diversos tipos de estudo de revisão, optou-se pela metanálise, que consiste em uma análise estatística dos resultados de diferentes estudos individuais, fazendo uma combinação destes para obter uma estimativa global do fenômeno avaliado (Mancini \& Sampaio, 2013).

\subsection{Critérios de inclusão}

Foram incluídos como referências artigos indexados em português e inglês, disponíveis em todos os periódicos do portal de periódicos CAPES, publicados em qualquer período. Foram localizados 263 artigos a partir da combinação dos descritores relacionados:

- "Religiosidade" + "qualidade de vida" = 121 documentos;

- "Espiritualidade" + "qualidade de vida" = 142 documentos.

\subsection{Critérios de exclusão}

Foram excluídos: monografias, dissertações e teses; os que constavam apenas em resumos; os que tratassem de 
religiosidade/espiritualidade e qualidade de vida, sem, entretanto, analisar a correlação entre ambas; e os que citassem os descritores, mas que esses não fossem o foco do estudo.

\subsection{Seleção de estudos}

Foi feita a leitura dos trabalhos encontrados na pesquisa para verificar se preenchiam os critérios de inclusão. Os que se adequaram aos critérios tiveram seus conteúdos analisados para a síntese de suas colaborações. Já os trabalhos que não satisfizeram os critérios de inclusão foram listados entre os excluídos. Após esse processo, dos 263 trabalhos localizados, 14 foram selecionados para o presente estudo.

\section{Resultados}

Foram selecionados 14 artigos que correspondiam aos critérios de inclusão. Dentre estes, o mais antigo tem como título "Saúde mental, qualidade de vida e religião em idosos de um Programa de Saúde da Família" (Floriano \& Dalgalarrondo, 2005); e os mais recentes são "Impacto da morte do filho sobre a conjugalidade dos pais" (Morelli, Scorsolini-Comin, \& Santos, 2013). e "Qualidade de vida e espiritualidade em seis doenças crônicas" (Meneses et al., 2013). (ver Tabela 1). 
Tabela 1

Distribuição dos artigos estudados segundo o título, autoria, ano de publicação e nome do periódico. Fortaleza/CE, 2013

\begin{tabular}{|c|c|c|c|}
\hline $\mathbf{N}$ & Título do Artigo & Autor & Ano \\
\hline 1 & $\begin{array}{l}\text { Saúde mental, qualidade de vida e } \\
\text { religião em idosos de um Programa de } \\
\text { Saúde da Familia }\end{array}$ & $\begin{array}{l}\text { Petterson de } \\
\text { Jesus Floriano e } \\
\text { Paulo } \\
\text { Dalgalarrondo }\end{array}$ & 2007 \\
\hline 2 & $\begin{array}{l}\text { Qualidade de vida e bem-estar espiritual } \\
\text { em universitários de psicologia }\end{array}$ & $\begin{array}{l}\text { Cristine Cardo } \\
\text { da Costa et al. }\end{array}$ & 2008 \\
\hline 3 & $\begin{array}{l}\text { Qualidade de vida e bem-estar espiritual } \\
\text { em pessoas vivendo com HIV/AIDS }\end{array}$ & $\begin{array}{l}\text { Prisla Ucker } \\
\text { Calvetti et al. }\end{array}$ & 2008 \\
\hline 4 & $\begin{array}{l}\text { Qualidade de vida e bem-estar espiritual } \\
\text { em pacientes com doença pulmonar } \\
\text { obstrutiva crônica }\end{array}$ & $\begin{array}{l}\text { Maira Shiramizu } \\
\text { da Silva et al. }\end{array}$ & 2009 \\
\hline 5 & $\begin{array}{l}\text { A terapia comunitária como um novo } \\
\text { instrumento de cuidado para saúde } \\
\text { mental do idoso }\end{array}$ & $\begin{array}{l}\text { Ianine Alves da } \\
\text { Rocha et al. }\end{array}$ & 2009 \\
\hline 6 & $\begin{array}{l}\text { Avaliação da espiritualidade dos } \\
\text { sobreviventes de cancro: implicações na } \\
\text { qualidade de vida }\end{array}$ & $\begin{array}{l}\text { Cândida Pinto e } \\
\text { José Luis Pais } \\
\text { Ribeiro }\end{array}$ & 2010 \\
\hline 7 & $\begin{array}{l}\text { Religiosidade/Espiritualidade em } \\
\text { Pacientes Oncológicos: Qualidade de } \\
\text { Vida e Saúde }\end{array}$ & $\begin{array}{l}\text { Silvia Aparecida } \\
\text { Fornazari e } \\
\text { Renatha El } \\
\text { Rafihi Ferreira }\end{array}$ & 2010 \\
\hline 8 & $\begin{array}{l}\text { Os sentidos da corporeidade em } \\
\text { ostomizados por câncer }\end{array}$ & $\begin{array}{l}\text { Suellen Santos } \\
\text { Lima de Almeida } \\
\text { et al. }\end{array}$ & 2010 \\
\hline 9 & $\begin{array}{l}\text { Qualidade de vida de mulheres vivendo } \\
\text { com o HIV/aids de um municipio do } \\
\text { interior paulista }\end{array}$ & $\begin{array}{l}\text { Joice Gaspar et } \\
\text { al. }\end{array}$ & 2011 \\
\hline $\begin{array}{l}1 \\
0\end{array}$ & $\begin{array}{l}\text { Cuidador familiar do idoso em cuidados } \\
\text { paliativos: o processo de morrer no } \\
\text { domicilio }\end{array}$ & $\begin{array}{l}\text { Flavia Renata } \\
\text { Fratezi e Beatriz } \\
\text { Aparecida Ozello } \\
\text { Gutierrez }\end{array}$ & 2011 \\
\hline $\begin{array}{l}1 \\
1\end{array}$ & $\begin{array}{l}\text { Avaliação de qualidade de vida e } \\
\text { importância dada a } \\
\text { espiritualidade/religiosidade/crenças } \\
\text { pessoais (SRPB) em adultos com e sem } \\
\text { problemas crônicos de saúde }\end{array}$ & $\begin{array}{l}\text { Neusa Sica da } \\
\text { Rocha e Marcelo } \\
\text { Pio da Almeida } \\
\text { Fleck }\end{array}$ & 2011 \\
\hline $\begin{array}{l}1 \\
2\end{array}$ & $\begin{array}{l}\text { Depressão em adolescentes praticantes } \\
\text { de caminhada }\end{array}$ & $\begin{array}{l}\text { Maria Christina } \\
\text { Soares Gomes } \\
\text { et al. }\end{array}$ & 2011 \\
\hline 3 & $\begin{array}{l}\text { Impacto da morte do filho sobre a } \\
\text { conjugalidade dos pais }\end{array}$ & $\begin{array}{l}\text { Ana Bárbara } \\
\text { Morelli et al. }\end{array}$ & 2013 \\
\hline $\begin{array}{l}1 \\
4\end{array}$ & $\begin{array}{l}\text { Qualidade de vida e espiritualidade em } \\
\text { seis doenças crônicas }\end{array}$ & $\begin{array}{l}\text { Rute F. Meneses } \\
\text { et al. }\end{array}$ & 2013 \\
\hline
\end{tabular}

Todos os artigos foram publicados em língua portuguesa, não sendo aproveitado nenhum artigo em língua inglesa. Percebe-se também que há um relativo aumento de publicações ao longo dos anos, com 
exceção do ano 2012 que não apresenta nenhuma publicação (ver Figura 1).

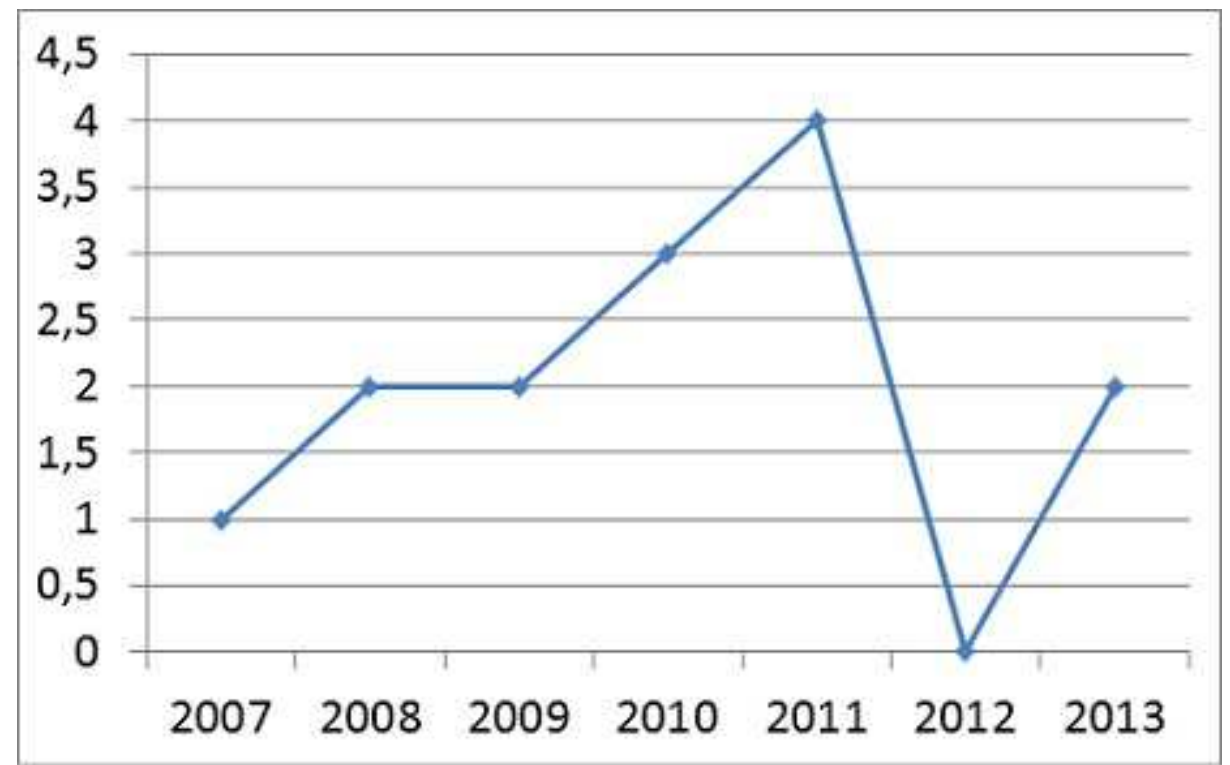

Figura 1. Número de trabalhos segundo ano de publicação. Fortaleza/CE, 2013

Destaca-se também que os artigos foram publicados em periódicos de diferentes áreas da saúde: Psicologia (7), Enfermagem (4), Medicina (2) e Saúde Coletiva (2). Esse fator mostra que várias áreas da saúde demonstram necessidade de avaliar e pesquisar sobre esse tema. Destaca-se, todavia, que a partir dos critérios de busca e seleção descritos no método, diferentemente do esperado, não foram contemplados artigos de outras áreas, tais como Ciências da Religião (ver Tabela 2).

Tabela 2

Quantidade de trabalhos publicados por área de concentração. Fortaleza/CE, 2013

\begin{tabular}{lc} 
Fortaleza/CE, 2013 & \\
\hline \multicolumn{1}{c}{ Área de concentração } & Frequência \\
\hline Psicologia & 7 \\
\hline Enfermagem & 3 \\
\hline Medicina & 2 \\
\hline Saúde Coletiva & 2 \\
\hline Total & $\mathbf{1 4}$ \\
\hline
\end{tabular}

A partir da leitura exaustiva dos artigos selecionados, foram sistematizadas categorias para a análise de acordo com a similaridade dos temas, facilitando a compreensão dos conteúdos apresentados, e permitindo a abordagem de divergências e convergências encontradas nos estudos. As categorias foram 
divididas em: 1) religiosidade e espiritualidade em pacientes com doenças crônicas, com 4 artigos; 2) importância da religiosidade para os idosos, com 2 artigos; 3 ) religiosidade e espiritualidade em pacientes com câncer, com 2 artigos; 4) religiosidade e espiritualidade em pacientes com HIV, com 2 artigos; 5) relação da religiosidade com a morte, com 2 artigos; 6) religiosidade em estudantes universitários, com 1 artigo; e 7) relação da religiosidade com a depressão, também com 1 artigo.

Referente à categoria 1, "Religiosidade e espiritualidade em pacientes com doenças crônicas", a partir da análise do "artigo 4" (Silva, Kimura, Stelmach, \& Santos, 2009), "artigo 6" (Pinto \& Ribeiro, 2010), "artigo 11" (Rocha \& Fleck, 2011) e "artigo 14" (Meneses et al., 2013), observou-se que as relações entre qualidade de vida e espiritualidade podem variar de acordo com a doença e com o doente, diferenciando-se de acordo com as peculiaridades da doença e subjetividade daquele que adoece e tem sua espiritualidade e qualidade de vida influenciando e sendo influenciada por essa nova condição. Contudo, de uma forma geral, pode-se concluir que a religiosidade e a espiritualidade apresentam consequências saudáveis para os pacientes com doenças crônicas.

No que se refere à categoria 2, "Importância da religiosidade para os idosos", a partir da análise do "artigo 1" (Floriano \& Dalgalarrondo, 2007) e do "artigo 5" (Rocha, Braga, Tavares, Andrade, Ferreira Filha, Dias, \& Silva, 2009), pode-se perceber que a religiosidade e a espiritualidade aparecem como um das estratégias mais utilizadas pelos idosos, apresentando uma relação saudável com a qualidade de vida dessa faixa etária.

Com relação à categoria 3, "Religiosidade e espiritualidade em pacientes com câncer", fundamentado no "artigo 7" (Fornazari \& Ferreira, 2010) e no "artigo 8" (Almeida et al., 2010), percebeu-se que a religiosidade e a espiritualidade aparecem novamente com relações saudáveis para a qualidade de vida dos pacientes com câncer, contribuindo até mesmo para a aceitação do tratamento.

Em se tratando da categoria 4, "Religiosidade e espiritualidade em pacientes com HIV", como resultado da análise do "artigo 3" (Calvetti, Muller, \& Nunes,2008) e do "artigo 9" (Gaspar, Reis, Pereira, Neves, Castrighini, \& Gir, 2011) pode-se observar que a religiosidade e a espiritualidade apresentam consequências positivas para a qualidade dos portadores de HIV, sendo utilizadas como fonte de apoio e aceitação.

No que concerne à categoria 5 , "Relação da religiosidade com a morte", a partir da análise do "artigo 10" (Fratezi \& Gutierrez, 2011) e do "artigo 13" (Morelli, Scorsolini-Comin, \& Santos, 2013), concluiu-se que, na relação com a morte, a religiosidade e a espiritualidade se mostram como estratégias de reflexão e 
enfrentamento, auxiliando os indivíduos a elaborar o luto e, consequentemente, a melhorar sua qualidade de vida.

Concernente a categoria 6, "Religiosidade em estudantes universitários", de acordo com o "artigo 2" (Costa, Bastiani, Geyer, Calvetti, Muller, \& Moraes, 2008), foi possível tomar como resultados que a dimensão espiritual não pode ser excluída da formação acadêmica, tanto por sua relevância quanto pelos prejuízos que o mau uso e o não entendimento possam causar. Ressaltaram também a importância de resgatar essa outra dimensão no atendimento à saúde das pessoas.

Por último, referente à categoria 7, "Relação da religiosidade com a depressão", com base no "artigo 12" (Gomes, Tolentino, Tolentino, Ramos, \& Durães, 2012), pode-se inferir que a religiosidade se mostrou importante para que o adolescente desse mais importância a ele mesmo. Além disso, mencionaram que a religiosidade é um fator protetor a ser considerado e também atuava como apoio na estrutura familiar.

\section{Conclusões}

O presente estudo foi motivado por várias indagações: $O$ comportamento religioso gera maturidade emocional? A religiosidade contribui para a adaptabilidade do indivíduo? A religião é uma fonte de doenças ou ativa a saúde psicológica? Há relação entre a qualidade de vida (positiva ou negativa do sujeito) em diferentes circunstâncias e sua religiosidade ou espiritualidade? Procurou-se examinar se a religião ajuda - ou não - as pessoas a viverem mais satisfatoriamente, conforme definido pela cultura e imaginado pelo senso comum.

Sabe-se que, ao se falar sobre saúde, é imprescindível abordar o conceito de qualidade de vida, tendo em vista que a sua construção surge pela necessidade de uma concepção ampliada de saúde que englobe todas as dimensões envolvidas nesse processo. Ao se deparar com o sujeito nas práticas de saúde, deve-se ter o compromisso de utilizar meios humanizados para tal, buscando entender o que é saudável para ele e, consequentemente, oferecer espaço para a sua subjetividade. Entendendo que a religiosidade e a espiritualidade podem ser elementos constituintes da subjetividade dos indivíduos, é de suma importância que os profissionais de saúde saibam lidar com esses fenômenos de maneira adequada.

Apesar de fazerem parte do cotidiano, esses fenômenos, no meio acadêmico e científico, são menosprezados, principalmente pela falta de embasamento empírico. Por esse motivo, o presente estudo buscou revisar as evidências empíricas de investigações sobre a correlação entre religiosidade, espiritualidade e qualidade de vida. 
Esperava-se, com isso, compreender os meios utilizados para avaliar esses fenômenos bem como elucidar, de forma factual, a importância com que eles aparecem na vida dos indivíduos.

$\mathrm{Na}$ análise dos resultados dos 14 artigos que atenderam aos critérios de inclusão foi possível identificar correlação entre a religiosidade, a espiritualidade e a qualidade de vida. Na maioria dos artigos observou-se uma correlação positiva entre esses, onde a religiosidade/espiritualidade aparece como uma das estratégias utilizadas para o enfrentamento de situações adversas, como doenças físicas, transtornos mentais ou o luto, mostra-se, portanto como um auxílio para o coping, de conforto e bem-estar, um mecanismo de defesa, ou até mesmo de resignação. Além disso, aparece como uma ótima ferramenta de apoio social para esses sujeitos. A espiritualidade, entendida como um fenômeno mais abrangente, também aparece com resultados bastante significativos nos estudos, como uma forma de o indivíduo dar novos significados aos seus percalços e reorganizar suas experiências. Até mesmo em sujeitos que não são adeptos de nenhuma religião a espiritualidade aparece como uma dimensão importante, atrelada a questões existenciais significativas.

Em apenas dois estudos, pôde-se observar uma correlação negativa entre esses fenômenos, especificamente no caso da religiosidade. Nesses estudos, o comportamento religioso aparece como um meio pelo qual os indivíduos buscam melhorias de suas condições através da fé, mas que se frustram na medida em que elas não vão acontecendo. Dessa forma, a religiosidade aparece acompanhada de menores índices de qualidade de vida. Outro fator a ser considerado, é que os dogmas e preceitos religiosos podem inibir o sujeito em lidar com determinados sentimentos, gerando mal-estar, ao invés de bemestar. Vale ressaltar, no entanto, que esses aspectos negativos apareceram em pequeno número e com índices muito baixos.

A literatura mostra que a relação entre a religião e a saúde é inconsistente e ambígua, podendo a fé, ou a religião, promover ou desencorajar bem-estar psicológico (Farris \& Rosa, 2011). Reconhece-se, pois, que "o compromisso religioso é um fenômeno multidimensional que é clinicamente pertinente e tem o potencial para beneficiar, frequentemente, ou prejudicar, ocasionalmente, a saúde mental" (Larson, Pattison, Blazer, Omran, \& Kaplan, 1996).

Reconhecem-se três tendências ou conclusões gerais: a religião é benéfica à saúde mental; há alguns tipos de religião que podem prejudicar a saúde; e religião não é a expressão das doenças mentais. (Farris \& Rosa, 2011).

Conclui-se ao final dessa pesquisa que a religiosidade pode ser fator de saúde e de qualidade de vida, ou não, a depender das características sociais, culturais, subjetivas, de personalidade e de saúde do sujeito que a vivência, além de diversos outros aspectos 
conhecidos e desconhecidos. A espiritualidade tem o potencial de trazer serenidade e felicidade autêntica aos humanos, a depender de como é vivenciada pelo sujeito, não sendo, portanto, regra geral.

A partir do que foi exposto até aqui, não se pode desconsiderar os aspectos benéficos que a religiosidade e a espiritualidade têm indicado. Por isso é de fundamental importância repensar uma aproximação da formação profissional com esses fenômenos, entendendo que eles se constituem como aspectos sociais e culturais dos contextos nos quais os indivíduos estão inseridos.

Com isso, serão formados profissionais de saúde mais preparados para lidar com o humano e será possível também promover uma aproximação do meio transcendental com o científico, reconhecendo as diferenças e limitações de ambos. Sendo assim, o presente estudo se mostra relevante para diversos setores da sociedade.

É importante ressaltar ainda a escassez de material escrito para ser utilizado nesse estudo. Ressalta-se que de 263 artigos analisados, apenas 14 corresponderam aos critérios de inclusão. Esse fator demonstra mais uma vez a pouca importância que esse tema tem tido no meio acadêmico e científico. A pesquisa poderia apresentar resultados ainda mais expressivos e precisos se existissem mais materiais para serem analisados. Atesta-se assim a necessidade de mais pesquisas nessa área, no intuito de fortalecer o conhecimento teórico e embasar de forma mais eficaz as práticas profissionais em saúde.

\section{Referências}

Almeida, R. (2009). A Igreja Universal e seus demônios. São Paulo: Terceiro Nome.

Alminhana, L. O. \& Moreira-Almeida, A. (2009). Personalidade e religiosidade/espiritualidade. Revista de Psiquiatria Clínica, 36(4),153-161. doi: 10.1590/S0101-60832009000400005

Angerami-Camon, V. A. (2004). Espiritualidade e prática clínica. São Paulo: Thomson.

Angerami-Camon, V. A. (2008). Psicologia e Religião. CENGAGE Learning: São Paulo.

Antoniazzi, A. (2003). As Religiões no Brasil Segundo o Censo de 2000. Revista Rever, (2), 75-80.

Barricelli, I. L. F. O. B. L., Sakumoto, I. K. Y., Silva, L. H. M., \& Araújo, C. V. (2012). Influência da orientação religiosa na qualidade de vida de idosos ativos. Revista Brasileira de Geriatria e Gerontologia, 15(3), 505-515. doi: 10.1590/S180998232012000300011 
Calvetti, P. Ü., Muller, M. C., \& Nunes, M. L. T. (2008). Qualidade de vida e bem-estar espiritual em pessoas vivendo com HIV/AIDS. Psicologia em Estudo, Maringá, 13(3), 523-530.

Costa, C. C., Bastiani, M., Geyer, J. G., Calvetti, P. Ü., Muller, M. C., \& Moraes, M. L. A. (2008). Qualidade de vida e bem-estar espiritual em universitários de psicologia. Psicologia em Estudo, Maringá, 13(2), 249-255.

Farris, R., Rosa, R. S. (2011). Religião salugênica e religião patogênica: uma aproximação à luz da psicologia. Revista Pistis \& Praxis, Teologia e Pastoral, 3(2), 361-382.

Fleck, A. P. M. [Org] (2008). A Avaliação de Qualidade de Vida: Guia para Profissionais da Saúde. $1^{\text {a }}$ ed. Porto Alegre: Artmed.

Fleck, M. P. A., Borges, Z. N., Bolognesi, G., \& Rocha, N. S. (2003). Desenvolvimento do WHOQOL, módulo espiritualidade, religiosidade e crenças pessoais. Revista de Saúde Pública, $37(4), 446-455$.

Floriano, P. J., \& Dalgalarrondo, P. (2007). Saúde mental, qualidade de vida e religião em idosos de um Programa de Saúde da Família. Jornal Brasileiro de Psiquiatria, 56(3), 162-170.

Fornazari, S. A., Ferreira, R. R. (2010). Religiosidade/espiritualidade em pacientes oncológicos: qualidade de vida e saúde. Revista Psicologia: Teoria e Pesquisa, 26(2), 265-272.

Fratezzi, F. R., \& Gutierrez, B. A. O. (2011). Cuidador familiar do idoso em cuidados paliativos: o processo de morrer no domicílio. Ciência \& Saúde Coletiva, 16(7), 3241-3248.

Gaspar, J., Reis, R. K., Pereira, F. M. V., Neves, L. A. S., Castrighini, C. C., \& Gir, E. (2011). Qualidade de vida de mulheres vivendo com o HIV/aids de um município do interior paulista. Revista Escola de Enfermagem USP, 45(1): 230-236.

Gomes, M. C. S., Tolentino, T. M., Tolentino, F. M., Ramos, C. A. R. \& Durães, M. F. G. (2012). Depressão em adolescentes praticantes de caminhada. Motricidade FTCD/FIP-MOC, 8(S2), 1051-1054.

James, W. (1985). The varieties of religious experience. Cambridge: Harvard University Press.

Kluthcovsky, A. C. G. C., \& Takayanagui, A. M. M. (2007). Qualidade de vida: aspectos conceituais. Revista Salus-Guarapuava-PR, 1(1): 13-15.

Larson, D. B., Pattison, E. M., Blazer, D. G., Omran, A. R., \& Kaplan, B. H. (1986). Systematic analysis of research on religious variables in four major psychiatric journals, 1978-1982. American Journal of Psychiatry, 143(3), 329-334.

Mancini, M. C., \& Sampaio, R. F. (2006). Quando o objeto de estudo é a literatura: estudos de revisão. Revista brasileira de fisioterapia, 10(4), 0-00. doi: 10.1590/S141335552006000400001 
Medeiros, B. (2010). A Relação entre Religiosidade, Culpa e Avaliação de Qualidade de Vida no Contexto do HIV/AIDS (Dissertação de Mestrado não publicada). Universidade Federal da Paraíba, João Pessoa, PB.

Meneses, R. F., Pais-Ribeiro, J., Pedro, L., Silva, I., Cardoso, H., Mendonça, D., Vilhena, E., Martins, A., Silva, A. M. (2013). Qualidade de vida e espiritualidade em seis doenças crônicas. Livro de atas do VIII simpósio nacional de investigação em psicologia.

Minayo, M. C. (2000). Qualidade de vida e saúde: um debate necessário. Ciência \& Saúde Coletiva, 5(1), 7-18. doi: 10.1590/S0102-311X2004000200027

Morelli, A. B., Scorsolini-Comin, F., \& Santos, M. A. (2013). Impacto da morte do filho sobre a conjugalidade dos pais. Ciência \& Saúde Coletiva, 18(9), 2711-2720.

Motta, P. R., \& Júnior, A. R. (2011). Psicologia, religião e espiritualidade: considerações sobre a natureza humana e o sentido existencial. Revista Educação, 6, 2.

Murakami, R., Campos C. J. G. (2012). Religião e saúde mental: desafio de integrar a religiosidade ao cuidado com o paciente. Revista Brasileira de Enfermagem, 65(2), 361-7. doi: 10.1590/S0034-71672012000200024

Noronha, D. P., \& Ferreira, S. M. S. P. (2000). Revisões de Literatura. In: Campello, B. S., Condón, B. V., \& Kremer, J. M. (Orgs.). Fontes de informação para pesquisadores e profissionais. Belo Horizonte: UFMG.

Pinto, C., \& Ribeiro, J. L. P. (2010). Avaliação da espiritualidade dos sobreviventes de cancro: implicações na qualidade de vida. Revista Portuguesa de Saúde Pública, 28(1), 49-56.

Paschoal, S. M. P. (2001). Qualidade de vida do idoso: elaboração de um instrumento que privilegia sua opinião. (Dissertação de Mestrado não publicada). Faculdade de Saúde Pública, Universidade de São Paulo, São Paulo.

Rocha, I. A., Braga, L. A. V., Tavares, L. M., Andrade, F. B., Ferreira Filha, M. O., Dias, M. D., \& Silva, A. O. (2009). A terapia comunitária como um novo instrumento de cuidado para saúde mental do idoso. Revista Brasileira de Enfermagem, Brasília, 62(5), 687-94.

Rocha, N. S., \& Fleck, M. P. A. (2004) Religiosidade, Saúde e Qualidade de Vida: Revisão de Literatura. In: Teixeira, E. F. B.; Muller, M. C. \& Silva, J. D. T. (Orgs). Espiritualidade e Qualidade de Vida. Porto Alegre: EDIPUCRS.

Rocha, N. S., \& Fleck, M. P. A. (2011). Avaliação de qualidade de vida e importância dada a espiritualidade/religiosidade/crenças pessoais (SRPB) em adultos com e sem problemas crônicos de 
saúde. Revista de Psiquiatria Clínica, 38(1): 19-23. doi: 10.1590/S0101-60832011000100005

Sanchez, Z. M., \& Nappo, S. A. (2007). A religiosidade, a espiritualidade e o consumo de drogas. Revista de Psiquiatria Clínica, 34, supl 1, 73-81.

Sanchez, Z. V. D. M., \& Nappo, S. A. (2007). A religiosidade, a espiritualidade e o consumo de drogas psicotrópicas. Revista de Psiquiatria Clínica, 34: 73-81.

Segre, M., \& Ferraz, F. C. (1997). O conceito de saúde. Revista de Saúde Pública, São Paulo, 31, 3, 538-42.

Seidl, E. M. F., \& Zannon, C. M. L. C. (2004). Qualidade de vida e saúde: aspectos conceituais e metodológicos. Cad. Saúde Pública, 20(2): 580-588. doi: 10.1590/S0102$311 \times 2004000200027$

Silva, M. S., Kimura, M., Stelmach, R., \& Santos, V. L. C. G. (2009). Qualidade de vida e bem-estar espiritual em pacientes com doença pulmonar obstrutiva crônica. Revista da Escola de Enfermagem da USP, 43(spe2): 1187-1192. doi: 10.1590/S0080-62342009000600007

Stroppa, A., \& Moreira-Almeida, A. (2008). Religiosidade e saúde. Saúde e Espiritualidade: uma nova visão da medicina. Mauro Ivan Salgado \& Gilson Freire (Orgs.). Belo Horizonte: Inede.

\section{Endereço para correspondência \\ Cynthia de Freitas Melo Lins}

Universidade de Fortaleza

Programa de Pós-Graduação em Psicologia

Av. Washington Soares, 1321, Bloco N Sala N 13, Bairro Edson Queiroz, CEP 60811-341, Fortaleza - CE, Brasil

Endereço eletrônico: cf.melo@yahoo.com.br

\section{Israel Silva Sampaio}

Universidade Estadual do Ceará

Coordenação de Psicologia - Campus do Itaperi

Avenida Dr. Silas Muguba, 1700, Bloco P, CEP 60740-000, Fortaleza - CE, Brasil

Endereço eletrônico: Ilf.israel@yahoo.com

\section{Deborah Leite de Abreu Souza}

Universidade Estadual do Ceará

Coordenação de Psicologia - Campus do Itaperi

Avenida Dr. Silas Muguba, 1700, Bloco P, CEP 60740-000, Fortaleza - CE, Brasil

Endereço eletrônico: deborah_leite@outlook.com

Nilberto dos Santos Pinto

Universidade Estadual do Ceará

Coordenação de Psicologia - Campus do Itaperi

Avenida Dr. Silas Muguba, 1700, Bloco P, CEP 60740-000, Fortaleza - CE, Brasil

Endereço eletrônico: nilberto_sanctos@hotmail.com

Recebido em: $31 / 07 / 2014$

Reformulado em: 04/02/2015

Aceito para publicação em: 17/03/2015 
Cynthia de Freitas Melo, Israel Silva Sampaio, Deborah Leite de Abreu Souza, Nilberto dos Santos Pinto

\section{Notas}

* Doutora em Psicologia. Professora do Programa de Pós-Graduação da Universidade de Fortaleza (Ceará-Brasil).

** Graduado em Psicologia pela Universidade Estadual do Ceará (Ceará-Brasil).

*** Graduando em Psicologia pela Universidade Estadual do Ceará (Ceará-Brasil).

**** Graduando em Psicologia pela Universidade Estadual do Ceará (Ceará-Brasil). 